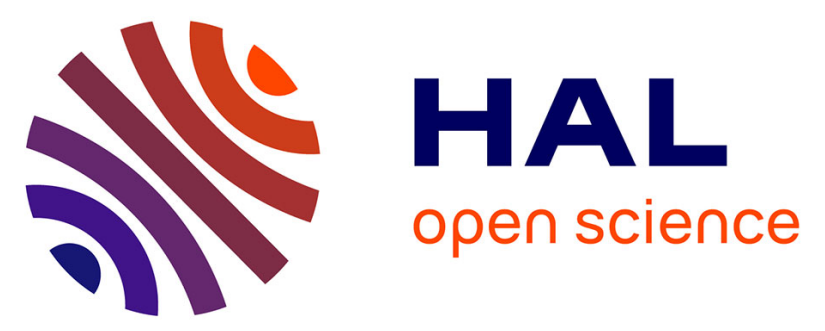

\title{
Temperature and orientation dependences of the E.S.R. linewidth in T.M.M.C.
}

\author{
G. Ablart, D. Bourdel, J. Pescia, S. Clement, J.P. Renard
}

\section{To cite this version:}

G. Ablart, D. Bourdel, J. Pescia, S. Clement, J.P. Renard. Temperature and orientation dependences of the E.S.R. linewidth in T.M.M.C.. Journal de Physique Lettres, 1981, 42 (10), pp.219-222. 10.1051/jphyslet:019810042010021900 . jpa-00231913

\section{HAL Id: jpa-00231913 https://hal.science/jpa-00231913}

Submitted on 1 Jan 1981

HAL is a multi-disciplinary open access archive for the deposit and dissemination of scientific research documents, whether they are published or not. The documents may come from teaching and research institutions in France or abroad, or from public or private research centers.
L'archive ouverte pluridisciplinaire HAL, est destinée au dépôt et à la diffusion de documents scientifiques de niveau recherche, publiés ou non, émanant des établissements d'enseignement et de recherche français ou étrangers, des laboratoires publics ou privés. 


\title{
Temperature and orientation dependences of the E.S.R. linewidth in T.M.M.C.
}

\author{
G. Ablart, D. Bourdel, J. Pescia \\ Laboratoire de Magnétisme et d'Electronique Quantique, 39 Allées Jules-Guesde, 31062 Toulouse cedex, France
}

\author{
S. Clément and J. P. Renard
}

Institut d'Electronique Fondamentale, Bâtiment 220, Université de Paris XI, 91405 Orsay cedex, France

(Reçu le 6 février 1981, accepté le 26 mars 1981)

\begin{abstract}
Résumé. - Nous présentons de nouveaux résultats sur la dépendance de la largeur de raie électronique du T.M.M.C. en fonction de l'orientation et de la température. Les données expérimentales sont en bon accord avec la théorie sur toute la gamme de températures utilisées ( 30 à $300 \mathrm{~K}$ ). Le comportement typiquement 1-d apparaît avec un cristal très pur et de préparation récente alors qu'on observe certains caractères 3-d après l'application de forts gradients de température.
\end{abstract}

\begin{abstract}
We present new data concerning the E.S.R. linewidth of T.M.M.C: as temperature and orientation are varied. The results obtained are in good agreement with theory in the whole temperature range investigated ( 30 to $300 \mathrm{~K}$ ). Typical 1-d behaviour is observed in a very pure, recently grown crystal while some 3-d characteristics occur after fast thermal changes.
\end{abstract}

1. Introduction. - Over the last decade, onedimensional magnetic compounds have been extensively investigated because experimental data obtained with them can easily be accounted for $[1,2]$. Among these materials, the tetramethyl ammonium manganese trichloride (T.M.M.C.) is the best one-dimensional system known at the present time. In effect, the ratio between its inter and intrachain exchange constants is as low as $10^{-4}$ [3]. Cheung et al. [4] and the present authors [5] have reported measurements of the linewidth in T.M.M.C. when temperature and the angle between the $c$-axis of the crystal and the magnetic field are varied. The plot of the linewidth versus temperature shows an anomalous behaviour about $20 \mathrm{~K}$, which we explained by assuming that a 3-d paramagnetic species is present in the crystal, in addition to the 1-d one. The 3-d species could be due to broken chains.

In this letter, we report new linewidth measurements at X-band with temperature ranging from 30 to $300 \mathrm{~K}$. We show that the anomalous temperature dependence disappears for very carefully prepared samples, protected from fast thermal changes. A correlation function with a Gaussian character at short times and a diffusive character at long times accounts well for the experimental data $[3,4]$. We also discuss the efficiency of the cut-off term commonly included in the diffusive part of the correlation function.

2. Apparatus. - The single crystal of T.M.M.C. was grown by slow evaporation of a saturated equimolar solution of $\mathrm{N}\left(\mathrm{CH}_{3}\right)_{4} \mathrm{Cl}$ and $\mathrm{MnCl}_{2}, 4 \mathrm{H}_{2} \mathrm{O}$. It was imbedded in polymethyl metacrylate. The sample was thus, protected from external damage and thermal changes. It was easily fixed on the rotating device used to determine angular dependences. Futhermore, the metacrylate does not produce any spurious E.S.R. signal. The magnetic purity of the sample was tested by analysing the E.S.R. line shape found using a modulation spectrometer [6]. In reference [5], we have shown how a 3-d-species could be discovered unambiguously in this way. The linewidths were measured using an homodyne spectrometer which detects the power reflected by the sample at resonance.

The temperature was varied from 4.2 to $300 \mathrm{~K}$, the sample being cooled in an E.S.R. 9 Oxford Instruments device. - The He-flow temperature was fixed by a heater and a temperature controller; the $\mathrm{He}$ flushed the single crystal, the temperature of which was carefully measured by a thermocouple fixed on the sample. 
3. Theoretical survey [4]. - In a very general form, the E.S.R. absorption can be expressed as [7] :

$$
I(\Delta \omega)=\frac{K^{\prime}(\Delta \omega)}{\pi\left[\left\{\Delta \omega-K^{\prime \prime}(\Delta \omega)\right\}^{2}+K^{\prime 2}(\Delta \omega)\right]}
$$

where $\Delta \omega=\omega-\omega_{z}$ and $\omega_{z}$ is the Larmor frequency.

The full half-power linewidth $\Delta H_{1 / 2}=2 \Delta \omega_{1 / 2} / \gamma_{\mathrm{e}}$ is then obtained from

$$
I(0) / 2=I\left(\Delta \omega_{1 / 2}\right)
$$

$K^{\prime}$ and $K^{\prime \prime}$ are given in terms of the memory function $\psi(t)$ :

$$
\begin{aligned}
K^{\prime}(\Delta \omega) & =\int_{0}^{\infty} \psi(t) \cos (\Delta \omega t) \mathrm{d} t \\
K^{\prime \prime}(\Delta \omega) & =\int_{0}^{\infty} \psi(t) \sin (\Delta \omega t) \mathrm{d} t .
\end{aligned}
$$

When the line is Lorentzian, the Kubo-Tomita results [8] are recovered :

$$
\Delta \omega_{1 / 2}=1 / T_{2}=K^{\prime}
$$

and the memory function can be identified with the «total-spin-torque » (TST) correlation function [3]

$$
\psi_{\mathrm{TST}}(t)=\frac{\left\langle\left[H^{\prime}(t), S^{+}\right]\left[S^{-}, H^{\prime}(0)\right]\right\rangle}{\left\langle S^{+} S^{-}\right\rangle}
$$

$H^{\prime}$ is the perturbation Hamiltonian responsible for the broadening. Here it corresponds to the intrachain dipolar interaction with a time evolution governed by the zero-order Hamiltonian : Exchange + Zeeman. $S^{+}$and $S^{-}$correspond to the transverse components of the total spin.

In 1-d magnetic substances, $\psi(t)$ is characterized by a long time diffusive decay [3] which is interrupted by the perturbing Hamiltonian $H^{\prime}$. It seems a good approximation to represent this behaviour by a phenomenological cut-off term related to the linewidth :

with

$$
\begin{aligned}
\psi(t) & =\psi_{\mathrm{TST}}(t) \mathrm{e}^{-\alpha t} \\
\alpha & =a \Delta \omega_{1 / 2} .
\end{aligned}
$$

The $a$ parameter allows the theoretical curves to be fitted with the experimental data. Its value is expected to be $\simeq 1$.

In the following calculation, it is convenient to separate $\psi(t)$ into a Gaussian short time part (until $\left.t=2 / \omega_{\mathrm{e}}\right)$ and a diffusive part :

$$
\psi(t)=\psi_{\mathrm{s}}(t)+\psi_{\mathrm{d}}(t) .
$$

Here, $\omega_{\mathrm{e}}$ is the exchange frequency, which is obtained from the first moments of the line

$\omega_{\mathrm{e}}=\left\{\frac{192 S(S+1)}{15}\right\}^{1 / 2} J / \hbar=9 \times 10^{12} \mathrm{rad} . / \mathrm{s} \gg \omega_{z}$.
From $\psi_{\mathrm{s}}(t)=\psi(0) \exp \left(-\omega_{\mathrm{e}}^{2} t^{2} / 2\right)$ a Lorentzian contribution $K_{\mathrm{S}}^{\prime}$ to the linewidth is drawn. The diffusive term can be written as

$$
\begin{aligned}
\psi_{\mathrm{d}}(t)=\left\{F_{0}^{2}(\theta)+F_{1}^{2}(\theta)\left(4 \mathrm{e}^{i \omega_{z} t}\right.\right. & \left.+6 \mathrm{e}^{-i \omega_{z} t}\right)+ \\
& \left.+F_{2}^{2}(\theta) \mathrm{e}^{-2 i \omega_{z} t}\right\} \phi(t)
\end{aligned}
$$

where the secular and the non-secular parts of the dipolar interaction have been explicitly considered.

$$
\begin{aligned}
& F_{0}^{2}(\theta)=A\left(1-3 \cos ^{2} \theta\right)^{2} \\
& F_{1}^{2}(\theta)=A \sin ^{2} \theta \cos ^{2} \theta \\
& F_{2}^{2}(\theta)=A \sin ^{4} \theta
\end{aligned}
$$

with

$$
\begin{gathered}
A=\frac{3 S(S+1)}{2} \omega_{\mathrm{D}}^{2} \zeta^{2}(3) \\
\omega_{\mathrm{D}}=\hbar \gamma_{\mathrm{e}}^{2} / c^{3}=9.7 \times 10^{9} \mathrm{rad} . / \mathrm{s}
\end{gathered}
$$

$c$ being the separation between two neighbouring $\mathrm{Mn}^{2+}$ on a chain

$$
\zeta(x)=\sum_{x} n^{-x} .
$$

When considering classical spins and taking the hydrodynamical limit, it can be shown that $\phi(t)$, which is a combination of four-spin correlation functions has indeed a diffusive behaviour. With the cutoff it becomes

$\phi(t)=(2 \pi D t)^{-1 / 2} \mathrm{e}^{-\alpha t} \quad D=6.9 \times 10^{12} \mathrm{rad} . / \mathrm{s} \quad[9]$.

Finally, one obtains

$$
K^{\prime}=K_{\mathrm{s}}^{\prime}+K_{\mathrm{d} 1}^{\prime}+K_{\mathrm{d} 2}^{\prime}+K_{\mathrm{d} 0}^{\prime}
$$

where the first three terms give a Lorentzian contribution to the linewidth :

$$
\begin{aligned}
K_{\mathrm{S}}^{\prime} & =3(\pi / 2)^{1 / 2} S(S+1)\left(\omega_{\mathrm{D}}^{2} / \omega_{\mathrm{e}}\right) \zeta(6)\left(1+\cos ^{2} \theta\right) \\
K_{\mathrm{d} 1}^{\prime} & =5 F_{1}^{2}(\theta)\left(D \omega_{\mathrm{z}}\right)^{-1 / 2}\left\{1-4\left(\omega_{z} / \pi \omega_{\mathrm{e}}\right)^{1 / 2}\right\} \\
K_{\mathrm{d} 2}^{\prime} & =1 / 2 F_{2}^{2}(\theta)\left(2 D \omega_{z}\right)^{-1 / 2}\left\{1-4\left(2 \omega_{z} / \pi \omega_{\mathrm{e}}\right)^{1 / 2}\right\} \\
K_{\mathrm{d} 0}^{\prime} & =F_{0}^{2}(\theta)(2 D)^{-1 / 2}(\cos \delta) /\left(\alpha^{2}+\Delta \omega^{2}\right)^{1 / 2}
\end{aligned}
$$

and

$$
K^{\prime \prime}=F_{0}^{2}(\theta)(2 D)^{-1 / 2}(\sin \delta) /\left(\alpha^{2}+\Delta \omega^{2}\right)^{1 / 2}
$$

where

$$
\cos 2 \delta=\alpha /\left(\alpha^{2}+\Delta \omega^{2}\right)^{1 / 2} .
$$

The temperature dependence of $\Delta H_{1 / 2}$ is obtained through the equation

$$
\psi(t, T)=\psi_{\mathrm{s}}(t, T=\infty) G_{\mathrm{s}}(T)+\psi_{\mathrm{d}}(t, T=\infty) G_{\mathrm{d}}(T)
$$

$G_{\mathrm{s}}(T)$, obtained from the temperature dependence of the moments of the line was computed by Tazuke and Nagata [10] using classical spins. In the $G_{\mathrm{d}}$ term, the 
temperature dependence of the diffusive constant [11] and the four-spin correlation functions are taken into account.

4. Discussion. - Firstly, we note an important result : the 3-d species discovered in reference [5], is not observed here. The data obtained with such a sample (hereafter referred to as the A-sample) are given in reference [5]. By comparing these with figure 1 , it can be inferred that the sample investigated in this paper (hereafter referred to as the B-sample) does not

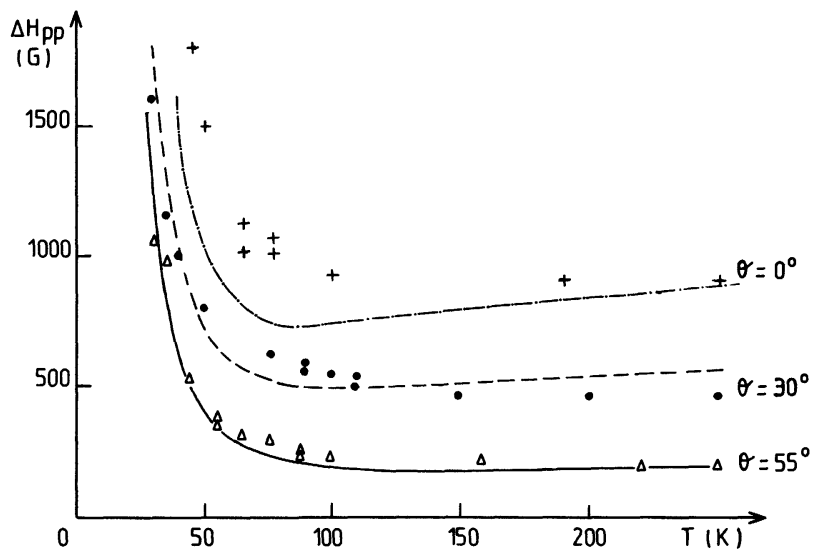

Fig. 1. - Temperature and angular dependences of the linewidth of a perfect T.M.M.C. crystal. The solid, dashed and dot-dashed lines are theoretical curves obtained from equation (1) with $a=1.6$ (Eq. (4)).

include a 3-d species. A study of the linewidth carried out with the modulation spectrometer confirms it : when the A-sample is used, we observe an isotropic two-peaked-resonance-line. With the B-sample, the line presents one peak only and disappears below $30 \mathrm{~K}$. It is very anisotropic which is typical of a 1-d system (Fig. 2). However, after fast temperature changes of the sample, the A-species features are again observed. So, we can conclude the 3-d species is due to broken chains.

Secondly, when comparing theory to experiment the agreement is satisfactory within a 20 pc error (the agreement is not so fair when using the data obtained by Soos et al. \{ Fig. 3 in Ref. [4] \}). The small discrepancy observed between 100 and $300 \mathrm{~K}$ seems to be due to the cut-off function : in effect, it has been introduced to limit diffusion and to insure the convergence of the $K^{\prime}(\Delta \omega)$ and $K^{\prime \prime}(\Delta \omega)$ integrals in equation (2). Two confirmations can be given : (i) at the magic angle $\left(\theta=54^{\circ}\right)$, the cut-off does not occur because the Zeeman modulation insures the convergence of the $K^{\prime}(\Delta \omega)$ and $K^{\prime \prime}(\Delta \omega)$ integrals. So, experi-

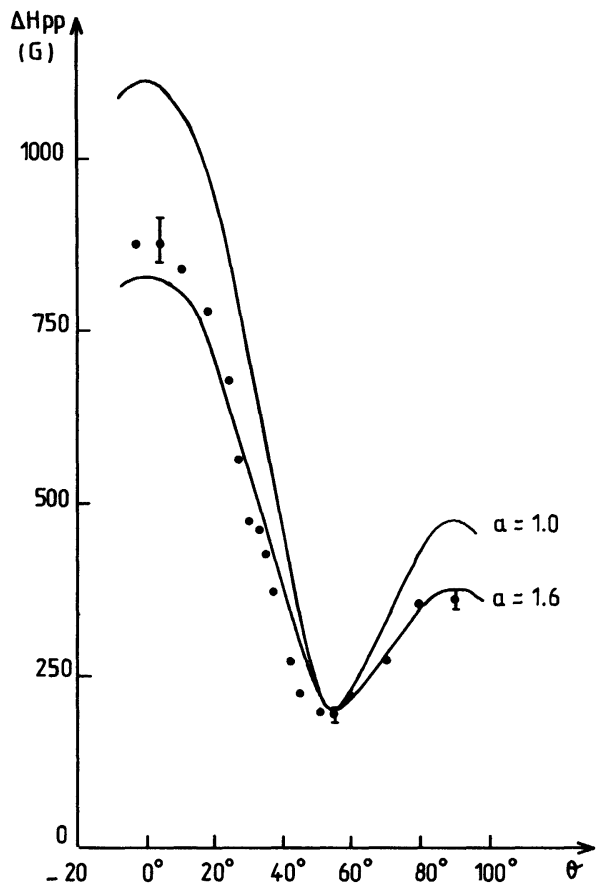

Fig. 2. - Angular dependence of the peak to peak derivative linewidth $\Delta H_{\mathrm{pp}}$ at $298 \mathrm{~K}$ and $\mathrm{X}$-band. The solid lines are theoretical curves obtained from equation (5) with two different $a$-values (Eq. (4)).

ment is seen to be in good agreement with theory. In contrast, when the angle is decreased from 54 to $0^{\circ}$, the difference between the experimental and theoretical curves is increased : in effect, at $0^{\circ}$ the linewidth is due only to the secular part of the dipolar Hamiltonian (the non-secular part being zero) and the cut-off is quite effective. (ii) Our $T_{1}$-measurements already published [12] are in fair agreement with theory : in effect, the cut-off is not necessary to limit diffusion [13] in the longitudinal relaxation.

5. Conclusion. - From the experimental temperature and orientation dependences of the E.S.R. linewidth, the following conclusions can be drawn : (i) the B-sample (carefully and recently grown and protected from thermal gradients) only shows 1-d magnetic properties. After fast thermal changes, both $1-d$ and 3-d behaviours are encountered [5]. Thus, the 3-d species seems to be due to thermally broken chains. (ii) From comparing the data found at $54^{\circ}$ to those obtained at other angles, the cut-off function used is seen to account for experiment within an error of $20 \%$. This is lowered at the magic angle, since the cut-off becomes inefficient. So, although this function does not seem to be the best one, at the present time a better model has not yet been proposed.

\section{References}

[1] Hone, D. W. and Richards, P. M., Ann. Rev. Mater. Sci. 4 (1974) 337.
[2] Steiner, M., Villain, J. and Windsor, C. G., Adv. Phys. 25 (1976) 87. 
[3] Boucher, J. P., Ahmed-Bakheit, M., Nechtschein, M., Villa, M., Bonera, G. and Borsa, F., Phys. Rev. B 13 (1976) 4098.

[4] Cheung, T. T. P., Soos, Z. G., Dietz, R. E. and Merrit, F. R Phys. Rev. B 17 (1978) 1266.

[5] Bourdel, D., Ruiz, F., Ablart, G., Pescia, J., Clement, S. and Renard, J. P., Solid State Commun. 29 (1979) 727.

[6] Ablart, G. and Pescia, J., Phys. Rev. B 22 (1980) 1150.

[7] Lado, F., Memory, J. D. and Parker, G. W., Phys. Rev. B 4 (1971) 1406.
[8] KUBo, R. and Tomita, K., J. Phys. Soc. Japan 9 (1954) 888.

[9] Lurie, N. A., Huber, D. L. and Blume, M., Phys. Rev. B 9 (1974) 2171.

[10] Nagata, K. and Tazuke, Y., J. Phys. Soc. Japan 38 (1975) 1003.

[11] Mori, M. and Kawasaki, K., Prog. Theor. Phys. 27 (1962) 529.

[12] Bourdel, D., Ablart, G., Pescia, J., Clement, S. and Renard, J. P., Phys. Rev. B 23 (1981) 1339.

[13] Bourdel, D., Thèse d'Etat, Toulouse (1980), unpublished. 